\title{
Ternary Dialectical Informatics
}

\author{
Nikolay Petrovich Brusentsov and Julia Sergeevna Vladimirova \\ Moscow State University \\ \{ramil, vladimirova\} @cs.msu.su
}

\begin{abstract}
Modern binary informatics with its so-called "classical" two-valued logic admits to create an artificial intellect and suppresses the natural intellect of students and other thinking people. Logic that based on dogmatic law of the excluded middle is incompatible with dialectical principle of opposition coexistence. Such logic is deprived of fundamental logical relation - the content consequence, and then cannot reach a conclusion. Aristotle's syllogistics includes the content consequence as common affirmative premise "All x are y". However, binarity misinterprets it as a paradoxical material implication that is not a relation at all. Lewis Carroll's "Symbolic logic" correctly represented syllogistics, but it is not intelligible for modern binary logic. Our paper reveals the essence of content consequence; it explains Carroll's intentional judgments and syllogistic relations submitted to opposite coexistence.
\end{abstract}

Keywords: Ternary informatics, opposition coexistence, content consequence, Aristotle's syllogistics, Carroll's "Symbolic Logic".

\section{Introduction}

The deficiency of modern binary informatics is specified with inadequacy of its foundation is adequate for its specification since it uses binary logic. This so-called "classical" formal logic, based on prior transcendental law of the excluded middle, does not show the perfect reflection of the reality and it is out of keeping with common sense. It is so opposite to practical arithmetic, for example, it is not useful for solving real problems. This binary logic is the logic of an artificial, discrete world of binary computers. There is no modality; possibility cannot differentiate from necessity. Even the most fundamental logical relation of content consequence has expressions by "material implication". These paradoxes for which distinguished logicians have tried for many years to overcome in vain.

It is clear why logic cannot become a school subject. The development of logical thinking or the study of logic, first as school task and then as a university one, does not lead to mentality improvement. The situation became worse with computerization of the education. With the general discussions concerning the intellectualization of computer processing of information and information safety, there is a suppression of people's intellect everywhere with stilted binary logic.

Most agree that Aristotle is the founder of logic. He created a system of demonstrative conclusions - the syllogistics that are still an unsurpassed mentality 
instrument. Syllogistics is dialectic; there is no paradox in it, but we cannot transform it into a modern logical calculus. This motivated suspicions that there was something wrong in Aristotle's logic such as his denial of empty sets. However, there are not only empty sets in syllogistics but also fuzzy sets that were invented by L. Zadeh in 1965 and these are still not "mastered" with modern logic. The principal difference of Aristotle's logic from modern, "classical" one is that it is not binary; rather it is ternary. Contrary to the "law of excluded middle" with "necessary is" and "necessary isn't", there is the third one affirming, "possible is and possible isn't". We predicate the "threevaluedness" to the relation of consequence that depletes the determined by Aristotle in the "Prior Analytics" [paragraph 57 line number 1]:

"...when two things are so related to one another, that if the one is, the other necessarily is, then if the latter is not, the former will not be either, but if the latter is, it is not necessary that the former should be. But it is impossible that the same thing should be necessitated by the being and by the not-being of the same thing"

\section{Mathematical Considerations}

In syllogistics, we present the consequence relation as universal affirmatives with premise "any $x$ is $y$ ". In this case, we see that any $x$-thing is necessarily a $x y$-thing, and any $y^{\prime}$-thing (not $y$-thing) is necessarily an $x^{\prime} y^{\prime}$-thing. Besides, a $x y^{\prime}$-thing must be an exclusion as $x$ must necessarily be $y\left(x\right.$ cannot be $\left.y^{\prime}\right)$. At the same time, we cannot exclude $x$ 'y-things; they are possible but not necessarily, as there are $x y$-things and $x^{\prime} y^{\prime}$-things. If we assume both premises, - "any $x$ is $y$ " and "any $y$ is $x$ "-then we must exclude $x y^{\prime}$ - and $x^{\prime} y$-things. Hence, there will exist an equivalence relation- " $x$ is interchangeable to $y$ ". In binary logic, we would have to admit "interchangeable/not interchangeable".

For the relation of consequence, we need three values, which are "necessary is", "possible but not necessary", and "impossible". Therefore, there is not enough binary implication for the adequate expression of consequence. With $x y^{\prime}$-things, consequence is impossible; if there are no $x y^{\prime}$-things, then we cannot exclude consequence; otherwise, it is possible but not necessary. In case of inexistence of $x$-things or $y^{\prime}-$ things, the implication does not express any interconnection between terms and does not form a two-dimensional relation. In these cases, the other term can have any value independently.

To smooth away "paradoxes" of implication, it is enough to prevent these cases of the invariability of its terms. Hence, a strict implication of Lewis is an inexistence of $x y^{\prime}$-things; that is, $\mathrm{V}^{\prime} x y^{\prime}$ is paradoxically performed when $x$-things do not exist and $y^{\prime}$ things do not exist - or when $V^{\prime} x$ and $V^{\prime} y^{\prime}$. There would not be paradoxes if with $V^{\prime} x y^{\prime}$ we demand $\mathrm{V} x$ and $\mathrm{V} y^{\prime}$ - the existence of $x$-things and $y^{\prime}$-things. As the result, the implication of Lewis becomes the necessary consequence $\mathrm{V}^{\prime} \mathrm{V}^{\prime} x y^{\prime} \mathrm{V}^{\prime}$ [3]. In full normal form of this relation, $\mathrm{V} x y \mathrm{~V}^{\prime} x y^{\prime} \mathrm{V} x^{\prime} y^{\prime}$, is visibly ternary. Members $x y$ and $x^{\prime} y^{\prime}$ belong to concerned subset Cartesian product $\left\{x, x^{\prime}\right\} \times\left\{y, y^{\prime}\right\}$, member $x y^{\prime}$ antibelongs to it and member $x^{\prime} y$ is withheld. Withholding expresses the third variety of belonging 
that is possible, but not necessary. A subset that admits a possible belonging is fuzzy and a relation presented with it is ternary.

In the mathematical logic, deviation from Aristotle's opinion of the universal affirmative premise that "All $A$ is $B$ ", takes it from a content consequence to binary implication. Hilbert and Ackerman proved the deviation with mathematical application logic needs "where taking Aristotle's opinion as a foundation was pointless" [7, p.79]. They did not take into consideration that the logic lost the richness of content, thinking that their logical calculus "makes possible successful problem comprehension where simple content logical thinking is principally weak" [7, p.17].

Indeed, logic became mindless two or three thousand years ago. Ancient stoics sought after incredible abstraction and carried it out with the help of "propositions" subordinated to "law of excluded middle". That law allowed only two truth-values"true" and "false". Adequate to reality, Aristotle's syllogistics became a dead scholasticism with binary logic. Mathematical logic presented this "classic" thinking with strict algebraic forms evidently showed its inadequateness [8].

Stoics "compensated" for the lack of the consequence relation in their logic to implement conclusions according to the rules of modus ponens and modus tollens. In mathematical logic, we know that an implication is not a consequence. "The relation 'if $X$ then $Y$ ' should not be understood as a form for relation of foundation and consequence. On the contrary, the proposition $X \rightarrow Y$ is always true when $X$ is false or when $Y$ is true" [7, p. 20]. At the same time, however, in mathematical logic even its founders identify binary implication with ternary and with Aristotle's consequence as the first and the second one associated with the proposition "All $A$ are $B$ ". As a result, from mathematical logic point of view, they consider the perfect forms of syllogisms darapti, bamalip, felapton, fesapo to be false [7, p.79]. The syllogism of submission of quotient to common is rejected as according to the deviation from Aristotle's interpretation "Some $A$ is $B$ " is not a consequence from "All $A$ are $B$ ".

Yan Lukasevitch created a ternary modal logic in 1920 in his detailed book titled, "Aristotle's syllogistics from a modern formal logic point of view" [9]. He proved algebraically with the help of the identification of the ternary consequence the material implication of Aristotle's statement- "But it is impossible that the same thing should be necessitated by the being and by the not-being of the same thing" - is false. It is also false from the logical point of view that they did not obey the basic logical law - the law of identity. We cannot identify the proposition "All $A$ are $B$ " that expresses the consequence relation $B$ from $A$ with "No one $A$ isn't not- $B$ ", the help of which binary implication relation is presented in natural language.

The problem is that logic cannot be without a consequence relation that is in essence is ternary; furthermore, it does not exist in binary logic. We express the relation called implication like consequence with the same "If... then..." relation and the same " $\rightarrow$ " marker. It is not striking that we can interpret implication as consequence. However, if there is no logic without consequence then logic with implication instead of consequence is not logic at all! Then from inexistent things follows "whatever", from $2 \times 2=4$ is that "snow is white".

Defective binary logic ignores common sense. The result of its application does not meet with natural expectations. In the book by T. Oppenheimer [10], he proves 
the indisputable bad influence of computer education in American schools. The author insists on removing computers from schools; but it is hardly possible in current situation. The "root of all evil" is not with computers but with the primitive unnatural logic of the discrete binary world that students study. It blocks their ability to master the logic of real world. If there were natural logic in computers, the result of studying computers would be the opposite.

However, there is no adequate (dialectical) logic in all "science of thinking", even where it does not follow the "law of excluded middle" and focuses on invention of not binary logics. This invention is unsuccessful because it has a formal character. If they researched the essentials of the problem, then they would have found out that Aristotle's logic is ternary and that ternary is a necessary, but not a sufficient, condition for adequate logic. Aristotle's logic is adequate; hence, it makes no sense to invent non-Aristotle logics.

\section{Carroll Logic}

A good exception is "Symbolic Logic" by Lewis Carroll [6] that did not receive appropriate (like Aristotle's one) understanding and development. There is neither discontent "true" or "false" propositions nor "law of excluded middle". His logic researches propositions that expressed interconnections of things characterized with combinations of features (peculiarities).

"The Universe contains 'Things' ... Things have 'Attributes' ... Any Attribute, or any Set of Attributes, may be called an 'Adjunct' "

The proposition is considered as a natural language expression of a relation that connects adjuncts of things with terms $x, y, z, \ldots$. At the same time, the essence of Carroll's relation visually reflects his diagram and algebraic "index method" that formally allows obtaining a content conclusion from data opinions, if they exist.

Carroll's diagram is on the surface identified to Pirs' truth table used to identify Boolean functions. However, expressed with the diagram, we interpret it not as extensional (as class of things) but intentional as set of things or subset of Cartesian product of pairs of opposite adjuncts. Besides, the diagram cells obtain not one from two values, but one from three values; it could also obtain cells that have " 0 " or " 1 " and they permit empty cells. They denote nothingness of the belonging of things to the subset presented in the diagram. Nevertheless, Carroll understood value "1" as existence, value " 0 " as inexistence of a thing; empty cells belong to neither.

For example, Lewis' strict implication relation V' $x y^{\prime}$ at Carroll's two-term diagram only one value appears- " 0 " in the $x y$-cell. Carroll expressed this relation in three ways using a universal negatives proposition: "No one $x y$ ' exists" or "no one $x$ is $y$ "' or "No one $y^{\prime}$ is $x$ ". The universal affirmative proposition "All $x$ are $y$ " includes a particular affirmative in Carroll's "Some $x$ is essence $y$ " that is equal to existence of the proposition "Some $x y$ exist". Carroll called "All $x$ are $y$ " a double proposition that was equal to two opinions: "No one $x$ is $y^{\prime \prime}$ " and "Some $x$ is y"; that is, V' $x y$ 'V $x y$. In the diagram, " 0 " is in the cell $x y$ ' and " 1 " is in the cell $x y$. 


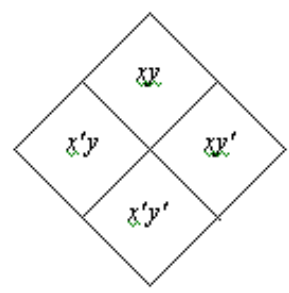

$x y \vee x^{\prime} y \vee x y^{t} \vee x^{\prime} y^{t}$

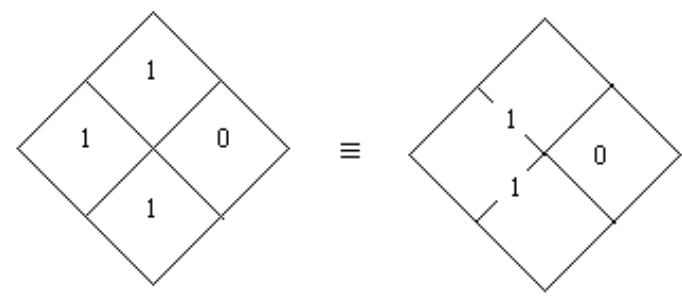

$x y \vee x^{t} y \vee x^{\prime} y^{t} \quad \equiv \quad x^{t} \vee y \equiv x \rightarrow y$

Fig. 1. Material Implication: Extensional interpretation (Pirs' table, Boolean class algebra)

\section{Mathematical Details}

It is clear that this is not implication (one of its paradoxes is eliminated) but it is not a full Aristotle consequence. He does not take into consideration the contraposition of a consequence - a false step, as it is peculiar to all efforts to make syllogism algebraic. As compensation [1], "Symbolic logic" of Carroll becomes a well-composed and perfect statement of Aristotle's syllogistics - a foundation of dialectical logic.

The most important component of logic content appeared an identified dialectic principle in the Aristotle's syllogistics - the principle of opposition coexistence [2]. It expresses the contraposition of a universal affirmative proposition as the symmetry of a relation expressed with a universal negative proposition; it constitutes the visible demonstrations of this principle. The fact is that the initial adjuncts $x, x^{\prime}, y, y^{\prime}, z, z^{\prime} \ldots$ expressed with terms $x, y, z \ldots$ made sense as the result of things comparison that have opposite adjuncts, for example, an $x$-thing and an $x^{\prime}$-thing. In other words, the principle of opposition coexistence means that a subset of the Cartesian product $\left\{x, x^{\prime}\right\} \times\left\{y, y^{\prime}\right\}$ reflects a content relation that has all pairs opposite adjuncts $\mathrm{V} x \mathrm{~V} x^{\prime} \mathrm{V} y \mathrm{~V}^{\prime}$. In Carroll's diagram, $\mathrm{V} x \mathrm{~V} x^{\prime} \mathrm{V} y \mathrm{~V} y^{\prime}$ reflected with token data of "1" existence on every one of four interior fences that denotes non-emptiness of classes $x$, $x^{\prime}, y, y^{\prime}$.

These equivalent realities are part of Aristotle's Universe (AU) - the foundation of content logic [2]. In it Lewis' implication V' $x y^{\prime}$ and Carroll's $V^{\prime} x V^{\prime} x y^{\prime}$ become a full consequence.

$$
\begin{aligned}
& \left(\mathrm{V}^{\prime} x y^{\prime}\right)\left(\mathrm{V} x \mathrm{~V} x^{\prime} \mathrm{V} y \mathrm{~V}^{\prime}\right) \equiv \mathrm{V} x y \mathrm{~V}^{\prime} x y^{\prime} \mathrm{V} x^{\prime} y^{\prime} \\
& \left(\mathrm{V} x \mathrm{~V}^{\prime} x y^{\prime}\right)\left(\mathrm{V} x \mathrm{~V} x^{\prime} \mathrm{V} y \mathrm{~V}^{\prime}\right) \equiv \mathrm{V} x y \mathrm{~V}^{\prime} x y^{\prime} \mathrm{V} x^{\prime} y
\end{aligned}
$$

Inexistence of any possible in the diagram things (e.g. an $x y$-thing in AU) means the existence of two adjoining things with it, so:

$$
\mathrm{V}^{\prime} x y^{\prime} \equiv\left(\mathrm{V}^{\prime} x y\right)\left(\mathrm{V} x \mathrm{~V} x^{\prime} \mathrm{V} y \mathrm{~V}^{\prime}\right) \equiv \mathrm{V}^{\prime} x y \mathrm{~V} x y^{\prime} \mathrm{V}^{\prime} y
$$




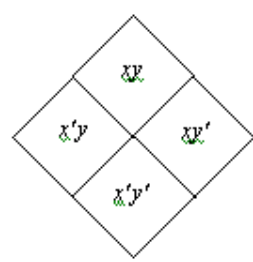

$\left\{x y, x y^{\prime}, x^{\prime} y, x^{\prime} y^{\prime}\right\}$

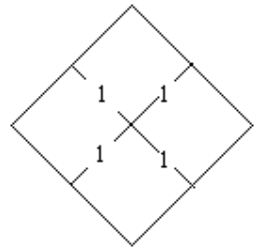

$\bigvee_{x} \bigvee_{x} \bigvee_{y} \bigvee_{y^{\prime}}$

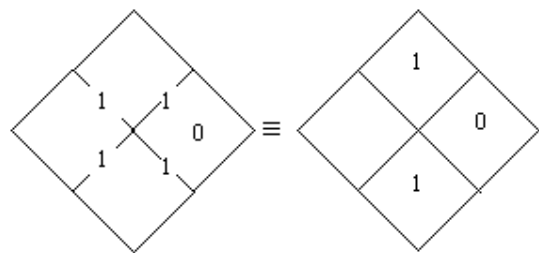

$\bigvee_{x} \bigvee_{x} \bigvee_{y} \bigvee_{y^{\prime}} \bigvee^{\prime} x y^{\prime} \equiv \bigvee_{x y} \bigvee^{\prime} x y^{\prime} \bigvee_{x^{\prime} y^{\prime}} \equiv(\mathrm{x} \Rightarrow \mathrm{y})$

Fig. 2. Content Consequence: Intentional interpretation (Carroll's diagram in Aristotle's Universe AU)

The existence of a $x y^{\prime}$-thing according to the principle of opposition coexistence entails the existence of its antipode - an $x^{\prime} y$-thing. Therefore, the particular affirmative and the particular negative premises of the syllogism become double and they are two, not four:

$$
\begin{aligned}
& \mathrm{I} x y \equiv \mathrm{A} x y \vee \mathrm{A} y x \equiv \mathrm{V} x y \mathrm{~V} x^{\prime} y^{\prime} \\
& \mathrm{O} x y \equiv \mathrm{E} x y \vee \mathrm{E} x^{\prime} y^{\prime} \equiv \mathrm{V} x y^{\prime} \mathrm{V} x y^{\prime}
\end{aligned}
$$

At the same time, common conditions are not two but four that come from one by term inverting:

$$
\mathrm{E} x y \equiv \mathrm{A} x y^{\prime}, \mathrm{E} x^{\prime} y^{\prime} \equiv \mathrm{A} x^{\prime} y, \mathrm{~A} y x \equiv \mathrm{A} x^{\prime} y^{\prime} .
$$

Syllogistics algebra that corresponds to Carroll's interpretation of its diagram with token symbolized existence and inexistence of things compared with cells and "fences" in similar to "index method" but instead of indexes it uses prefix functor of existence $\mathrm{V}$ - "disjunct" (integral disjunction similar to integral sum $\Sigma$ ) and its inversion $\mathrm{V}^{\prime}$ symbol of inexistence. The illustration presented in the diagram relation reflects a conjunction of disjuncts, not inverted and inverted, and members of conjunction that corresponds to empty cells that are not present (silent). Every trit takes one of three values: "+" for existence, "-" for inexistence, and "0" for silence. For example,

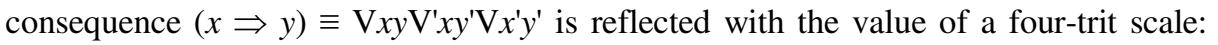
$+-0+$, a particular negative premise $\mathrm{O} x y$ coded with the value: $0++0$. There are eight double place relations in syllogistics [5]:

$$
\begin{aligned}
& \mathrm{A} x y \equiv \mathrm{A} y^{\prime} x^{\prime} \equiv \mathrm{E} x y^{\prime} \equiv \mathrm{E} y^{\prime} x \equiv+-0+ \\
& \mathrm{A} y x \equiv \mathrm{A} x^{\prime} y^{\prime} \equiv \mathrm{E} y x^{\prime} \equiv \mathrm{E} x^{\prime} y \equiv+0-+ \\
& \mathrm{E} x y \equiv \mathrm{E} y x \equiv \mathrm{A} x y^{\prime} \equiv \mathrm{A} y x^{\prime} \equiv-++0 \\
& \mathrm{E} x^{\prime} y^{\prime} \equiv \mathrm{E} y^{\prime} x^{\prime} \equiv \mathrm{A} x^{\prime} y \equiv \mathrm{A} y^{\prime} x \equiv 0++- \\
& \mathrm{I} x y \equiv \mathrm{I} x^{\prime} y^{\prime} \equiv \mathrm{I} y x \equiv \mathrm{I} y^{\prime} x^{\prime} \equiv \mathrm{O} x y^{\prime} \equiv \mathrm{O} y x^{\prime} \equiv \mathrm{O} x^{\prime} y \equiv \mathrm{O} y^{\prime} x \equiv+00+ \\
& \mathrm{O} x y \equiv \mathrm{O} y^{\prime} x^{\prime} \equiv \mathrm{O} y x \equiv \mathrm{O} x^{\prime} y^{\prime} \equiv \mathrm{I} x y^{\prime} \equiv \mathrm{I} y^{\prime} x \equiv \mathrm{I} x^{\prime} y \equiv \mathrm{I} y x^{\prime} \equiv 0++0 \\
& x \Leftrightarrow y \equiv \mathrm{A} x y \mathrm{~A} y x \equiv \mathrm{E} x y^{\prime} \mathrm{E} y x^{\prime} \equiv+--+ \\
& x \Leftrightarrow y^{\prime} \equiv \mathrm{E} x y \mathrm{E} x^{\prime} y^{\prime} \equiv \mathrm{A} x y^{\prime} \mathrm{A} y x^{\prime} \equiv-++-
\end{aligned}
$$

Computerizing proof of conclusions (true modi of syllogism) is carried out by two terms premise presentation with three term scales from witch crossing searching 
conclusion is taken by middle term elimination, if it exists. For example, modus Barbara: $\mathrm{A} y z \mathrm{~A} x y \Rightarrow \mathrm{A} x z$ in three term $x, y, z$-scales is realized:

$$
\begin{aligned}
& \text { Ayz } \equiv+-0++-0+ \\
& \text { Axy } \equiv++--00++ \\
& \text { Ayz } \cap \text { Axy } \equiv+---000+
\end{aligned}
$$

The elimination $y$ gives $x$, $z$-scale $+-0+$, i.e. Axz. Subordination of particular premises is proved with a common crossing of coding these premises scales. So, the subordination $\mathrm{A} x y \Rightarrow \mathrm{I} x y$, is equal to $\mathrm{A} x y \mathrm{I} x y=\mathrm{A} x y$, proved with crossing

$$
+-0+\bigcap+00+=+-0+\text {. }
$$

In syllogistics based on the opposition coexistence of all doubtful modi from the classical logic point of view and a row of modi missed with traditional syllogistics. For example, from premises of doubtful modus bamalip, there is not only particular but also a universal conclusion:

$$
\begin{aligned}
& \mathrm{A} z y \equiv+0-++0-+ \\
& \mathrm{A} y x \equiv++00--++ \\
& \mathrm{A} z y \cap \mathrm{A} y x \equiv+0-0---+
\end{aligned}
$$

Eliminating $y$ we have

$$
+0-+\equiv \mathrm{A} z x \equiv \mathrm{A} x^{\prime} z^{\prime}
$$

i.e., AzyAyx $\Rightarrow \mathrm{A} z x$

The correction of traditional theory is proof of denying with it modus of the first figure IyzAxy $\Rightarrow \mathrm{I} x z$ :

$$
\begin{aligned}
& \mathrm{I} y z \equiv+00++00+ \\
& \mathrm{A} x y \equiv++--00++ \\
& \mathrm{I} y z \cap \mathrm{A} x y \equiv+0--000+
\end{aligned}
$$

That with $y$ elimination is $+00+$, i.e. I $x z$. By the same way, the true of the next missed modus is proof of the first figure $\mathrm{I} y z \mathrm{E} x y \Rightarrow \mathrm{O} x z$ and similar modi of other figures.

\section{References}

[1] Brusentsov, N.P.: Diagrams of L. Carroll and Aristotle syllogistics. Computers and Problems of Cybernetics Questions (13), 164-182 (1977)

[2] Brusentsov, N.P.: Wandering around three pines (dialectics adventures in informatics) (2000), http: / / ternarycomp.cs.msu .ru/Papers / 3 PINES . pdf

[3] Brusentsov, N.P.: Ternary interpretation of Aristotle syllogistics. Historical Mathematical Researches 43(8), 317-327 (2003)

[4] Brusentsov, N.P., Vladimirova, J.S.: Ternary computerization of logic. In: 12th Russian Conference on Mathematical Methods of Form Identification, Report Digest, pp. 40-42 (2005)

[5] Brusentsov, N.P.: Reanimation of Aristotle's syllogistics. Logic Restoration, 140-145 (2005) 
[6] Carroll, L.: Symbolic logic. L. Carroll Story with Bundles, 189-361 (1973)

[7] Hilbert, D., Ackermann, W.: Foundations of Theoretical Logic (1947)

[8] Losev, A.F.: Critical notes about bourgeois mathematical logic. Historical Mathematical Researches 43(8), 339-401 (2003)

[9] Lukasevitch, Y.: Aristotle's Syllogistics from Modern Formal Logic Point of View (1959)

[10] Oppenheimer, T.: The Flickering Mind: The False Promise of Technology in the Classroom and How Learning Can Be Saved, p. 512. Random House, New York (2003) 